\title{
Discordance of PIK3CA and TP53 Mutations Between Breast Cancer Brain Metastases and Matched Primary Tumors
}

\section{ANNA THULIN}

Department of Oncology, Institute of Clinical Sciences, Sahlgrenska Academy at University of Gothenburg

\section{Carola Andersson}

Diagnostic Center, Kalmar, Regional hospital of Kalmar county

\section{Elisabeth Rönnerman}

Department of Clinical Pathology, Sahlgrenska University Hospital, Gothenburg

\section{Shahin Lara}

Department of Clinical Pathology, Sahlgrenska University Hospital, Gothenburg

\section{Chaido Chamalidou}

Department of Oncology, SKAS, Skövde

\section{Arnd Schoenfeldt}

Department of Pathology, Norra Älvsborg Hospital, Trollhättan

\section{Anikó Kovács}

Department of Clinical Pathology, Sahlgrenska University Hospital, Gothenburg

\section{Henrik Fagman}

Sahlgrenska Cancer Center, Department of Laboratory Medicine, Institute of Biomedicine, Sahlgrenska Academy at University of Gothenburg, Gothenburg

\section{Fredrik Enlund}

Diagnostic Center, Kalmar, Regional hospital of Kalmar county

\section{Barbro Linderholm ( $\sim$ barbro.linderholm@oncology.gu.se )}

Department of Oncology, Institute of Clinical Sciences, Sahlgrenska Academy at University of Gothenburg

\section{Research Article}

Keywords: Breast cancer brain metastases, next generation sequencing (NGS), PIK3CA, TP53

Posted Date: October 13th, 2021

DOl: https://doi.org/10.21203/rs.3.rs-525945/v2 
License: (c) (i) This work is licensed under a Creative Commons Attribution 4.0 International License. Read Full License

Version of Record: A version of this preprint was published at Scientific Reports on December 1st, 2021. See the published version at https://doi.org/10.1038/s41598-021-02903-x. 


\section{Abstract}

Purpose: There is limited knowledge of the biology of breast cancer (BC) brain metastasis (BM). We primarily aimed to determine the mutations in BCBM and to compare the mutational pattern with the matched primary breast cancer $(B C)$. Secondary aims were to determine mutations in each subgroup (Luminal, HER2+ and TNBC) of BCBM and to determine survival according to specific mutations.

Patients and methods: We investigated 57 BCBMs, including 46 cases with matched primary tumors (PT) by targeted Next Generation Sequencing (NGS) using the Cancer Hotspot Panel v2 (ThermoFisher Scientific) covering 207 targeted regions in 50 cancer related genes. Subtype according to immunohistochemistry was re-evaluated.

Results: NGS results fulfilling sequencing quality criteria were obtained from $52 \mathrm{BM}$ and $41 \mathrm{PT}$, out of which 37 were matched pairs. Pathogenic mutations were detected in $66 \%$ of PTs $(27 / 41)$, and $62 \%$ of BMs (32/52). TP53 mutations were most frequent; $49 \%$ (20/41) of PTs and $48 \%(25 / 52)$ in BMs, followed by PIK3CA mutations; 22\% (9/42) in PTs and 25\% (13/52) in BMs. Mutations in CDH1, EGFR, HRAS, RB1 $C D K N 2 A$ and $P T E N$ were detected in single pairs or single samples. Mutational pattern was discordant in $24 \%$ of matched pairs.

Conclusions: We show a discordance of PIK3CA and TP53 mutations of roughly $25 \%$ indicating the need to develop methods to assess mutational status in brain metastasis where analysis of cell-free DNA from cerebrospinal fluid (CSF) has shown promising results.

\section{Introduction}

Despite increasingly effective treatment, about $20 \%$ of patients with primary breast cancer (BC) suffer from metastatic disease and $15-40 \%$ of these patients eventually develop brain metastases (BM) [1]. Due to limited treatment options, and debilitating symptoms that greatly affect quality of life, BM is a dreaded outcome [2]. There is a large variation in survival after diagnosis of BM that ranges from Triple Negative Breast Cancer (TNBC), with a median survival of 4-5 months, to Human Epidermal growth factor Receptor 2 (HER2) positive (HER2+) BC with a median survival following BM diagnosis of 9-16 months $[3,4]$. The subtype of $B C$ influences the risk of developing BMs and the BM free interval, with significantly higher incidence and shorter interval from recurrence to diagnosis of BM for patients with TNBC and HER2 + as compared to luminal tumors $[5,6]$.

Local treatment such as surgery and/ or radiotherapy forms the basis of BM treatment. There is also clear evidence showing systemic therapies to have an effect on BM in patients ineligible for local treatment $[7,8]$. However, the blood-brain-barrier as well as the blood-tumor-barrier hinders the passage of systemic therapies to the central nervous system, hampering the effect of systemic treatment on BMs [9]. Another clinical problem is the difficulty to access metastatic tissue from the brain. Repeated studies have shown a discordance in the expression of steroid receptor and HER2 status between metastatic 
lesions and the primary BC [10]. A rapid development of new compounds targeting HER, CDK4/6, PARP and PIK3CA is underway. Hence, the need for analysis of BC tissue in the metastatic setting is apparent.

$\mathrm{BM}$ have become increasingly common in $\mathrm{BC}[6,11]$ and consequently, there is need for adequate information regarding the evolution of mutations, and histology in the metastatic process for correct therapy decisions [12-14]. There are studies investigating gene expression in metastatic lesions [15]. However, due to difficulties to sample tissue, BMs represent very few of investigated metastatic lesions.

We primarily aimed to determine the mutations in BCBMs and to compare the results with mutations in matched primary BC using next generation sequencing (NGS). Secondly, we aimed to investigate the mutations in each BC subgroup (Luminal, HER2 + and TNBC) and to determine survival according to specific mutations.

\section{Material And Methods}

\section{Patients}

From hospital records of all diagnostic codes, patients with BMs from BC between 1994 and 2014 were identified. Patients received treatment of the primary BC in any of four hospitals in the western region of Sweden. The diagnoses were confirmed in the patient's charts and patients with available material from BMs were selected. Clinical characteristics, type of metastasis, progression and survival were extracted from patient charts. PTs and BM tissue were evaluated, as specified below, when sufficient material was available. Data from the original report was utilized if deemed appropriated by the responsible breast pathologist if there was insufficient material available for re-evaluation by IHC. The study was conducted in accordance with the Declaration of Helsinki and the Sahlgrenska University Hospital Ethical Review Board; Gothenburg, Sweden approved the study (460-09, T592-14). Approval for the chart review and bio bank extractions was granted from each head of the participating departments.

\section{Immunohistochemistry (IHC)}

The available material was re-evaluated for histological type, nuclear grade, and receptor status. ER/PR, Ki67 and HER2 (HercepTest) immunohistochemistry was performed as per standard procedures using the Dako Autostainer Link and the EnVision ${ }^{\mathrm{TM}}$ FLEX detection systems according to the manufacturer's instructions. HercepTest was followed by SISH when the IHC was judged as $2+$ or $3+$. IHC was used to construct the subgroups §. Luminal A was defined as ER positive, PR present and/ or low Ki67. Luminal B was defined as ER positive, PR absent and/ or high Ki67. HER2+/ ER + was defined as amplified HER2 and ER positive. HER2+/ ER- was defined as HER2 amplified and ER negative. TNBC was defined as lacking HER2 amplification, ER positivity and PR positivity.

\section{Next Generation Sequencing}




\section{Preparation of sample library and next generation sequencing (NGS)}

DNA isolation from FFPE sections containing a minimum of $25 \%$ neoplastic cells as assessed by breast pathologists was performed using the QIAamp DNA FFPE tissue kit (Qiagen Gmbh, Hilden, Germany). DNA concentration was determined using NanoDrop ${ }^{\mathrm{TM}} 3300$ with Quant-IT Picogreen dsDNA assay kit (ThermoFisher Scientific, Waltham, MA, USA). Ten ng of DNA was used to prepare barcoded libraries with the Ion AmpliSeq ${ }^{\text {TM }}$ Library kit 2.0 (ThermoFisher). The Cancer Hotspot Panel v2 (ThermoFisher Scientific) covering 207 targeted regions in 50 cancer related genes was used (https://tools.lifetechnologies.com/content/sfs/brochures/lon-AmpliSeq-Cancer-Hotspot-Panel-Flyer.pdf). Template preparation and enrichment was performed with the lonChef ${ }^{\mathrm{TM}}$ Instrument (ThermoFisher Scientific). Eight barcoded samples were pooled per lon $318^{\mathrm{TM}} \mathrm{V} 2 \mathrm{BC}$ chip and sequenced on the lon $\mathrm{PGM}^{\mathrm{TM}}$ System (ThermoFisher Scientific). All steps were performed according to the manufacturer's instruction. Matched germline DNA from the patients was not available in this retrospective investigation.

\section{Data processing}

After alignment to the hg19 human reference genome, variant calling by the Torrent Suite Software v4.2.1.0 and filtering of described SNPs was performed. Variants were visually inspected with the Integrative Genomics Viewer (IGV; Broad Institute, Cambridge, MA, USA). Mutations were manually curated as pathogenic, likely pathogenic, variants of unknown significance or as benign. For ten samples, NGS results could not be obtained due to insufficient quality of DNA. For individual variants the minimum accepted read depth was 500 and if the read depth was $<1000$, an allele frequency of $>5 \%$ was required for positive variant calling.

\section{Statistical Methods}

Tests of differences between subgroups and mutations was performed using Chi-square test or Fischer Exact test, depending on the number of expected values, with $5 \%$ significance as the limit to reject the null hypothesis. Brain Metastasis Specific Survival (BMSS) i.e. time from diagnosis of BM to death were estimated by the Kaplan Meier estimator, and compared by the log rank test. Two patients who had not undergone surgery were removed from survival analyses. IBM SPSS Statistics $25^{\mathrm{TM}}$ was used for statistical calculations.

\section{Results}

After identifying 69 patients using diagnostic codes and charts, 46 patients remained with available matched PT and BM tissue. For 11 patients, only the BM tissue was available. Thus 57 BMs and 46 PTs were included in the study (Fig. 1). Of 57 patients with BM, 55 had undergone surgery (96\%). The median age at time of BM diagnosis was 53 (29-75) years (clinical data is specified in Table 1 and 2). The 22 
patients with extra-cranial disease (ED) prior to BM had a median time from ED to BM of 18.1 months (range 1.4-92 months).

Table 1

Clinical characteristics at primary breast cancer of the 46 patients with available primary tumor material

$\begin{array}{lllll}\text { All }(n= & \text { Luminal A } \\ 46) & (n=6) & \begin{array}{l}\text { Luminal B } \\ (n=6)\end{array} & \left.\begin{array}{l}\text { TNBC } \\ =23\end{array}\right) & \left.\begin{array}{l}\text { HER2+ } \\ =11\end{array}\right)\end{array}$

Age at diagnosis of $\mathrm{BC}$ (years)

$\begin{array}{llllll}\text { Mean (Standard Deviation (SD) } & \mathbf{4 9 ( 9 . 9 )} & 54(10.1) & 50(8.2) & 46(9.5) & 46(9.3) \\ \text { Median (min; max) } & \mathbf{5 1} & 58(40 ; 64) & 51(41 ; 60) & 54(32 ; & 45(33 ; \\ & \mathbf{( 3 2 ; 7 2 )} & & & 67) & 55)\end{array}$

\section{Stage at diagnosis of $\mathrm{BC}$}

\begin{tabular}{|c|c|c|c|c|c|}
\hline I & $7(15 \%)$ & $0(0 \%)$ & $2(33 \%)$ & $2(9 \%)$ & $3(27 \%)$ \\
\hline II & $\begin{array}{l}13 \\
(28 \%)\end{array}$ & $2(33 \%)$ & $2(33 \%)$ & $6(26 \%)$ & $3(27 \%)$ \\
\hline III & $\begin{array}{l}20 \\
(44 \%)\end{array}$ & $3(50 \%)$ & $2(33 \%)$ & $11(48 \%)$ & $4(36 \%)$ \\
\hline IV & $6(13 \%)$ & $1(17 \%)$ & 0 & $4(17 \%)$ & $1(9 \%)$ \\
\hline \multicolumn{6}{|l|}{ Histological Subtype } \\
\hline Ductal Invasive & $\begin{array}{l}42 \\
(91 \%)\end{array}$ & $6(100 \%)$ & $5(83 \%)$ & $22(96 \%)$ & 9 (82\%) \\
\hline Lobular Invasive & $3(7 \%)$ & $0(0 \%)$ & $0(0 \%)$ & $1(4 \%)$ & $2(18 \%)$ \\
\hline Other & $1(2 \%)$ & $0(0 \%)$ & $1(17 \%)$ & $0(0 \%)$ & 0 \\
\hline \multicolumn{6}{|l|}{ BRE Grade } \\
\hline II & $\begin{array}{l}12 \\
(26 \%)\end{array}$ & $2(33 \%)$ & $1(20 \%$ & $6(26 \%)$ & $3(27 \%)$ \\
\hline III & $\begin{array}{l}34 \\
(74 \%)\end{array}$ & $4(67 \%)$ & $5(80 \%)$ & $17(24 \%)$ & $8(73 \%)$ \\
\hline \multicolumn{6}{|c|}{$\begin{array}{l}\text { Brain Metastasis Free Interval } \\
\text { (months) }(n=46)\end{array}$} \\
\hline Mean (SD) & $\begin{array}{l}48 \\
(52.26)\end{array}$ & $58(45)$ & $74(21)$ & $39(38)$ & $63(34)$ \\
\hline Median (min; max) & $\begin{array}{l}51(11 ; \\
288)\end{array}$ & $49(0 ; 113)$ & $73(48 ; 97)$ & $\begin{array}{l}33(0 ; \\
196)\end{array}$ & $\begin{array}{l}51(34 \\
113)\end{array}$ \\
\hline
\end{tabular}


Table 2

Clinical data of the 57 patients with available brain metastasis

\begin{tabular}{|c|c|c|c|c|c|}
\hline & $\begin{array}{l}\text { All }(n= \\
57)\end{array}$ & $\begin{array}{l}\text { Luminal } \\
\text { A }\end{array}$ & $\begin{array}{l}\text { Luminal B ( } \mathrm{n} \\
=14)\end{array}$ & $\begin{array}{l}\text { TNBC }(n \\
=26)\end{array}$ & $\begin{array}{l}\text { HER2+(n } \\
=17)\end{array}$ \\
\hline \multicolumn{6}{|l|}{ Age at diagnosis of BM (years) } \\
\hline Mean (SD) & $\begin{array}{l}53 \\
(10.9)\end{array}$ & & $55(10)$ & $54(10.7)$ & $49(11)$ \\
\hline Median (min; max) & $\begin{array}{l}53(29 ; \\
75)\end{array}$ & & $55(40 ; 70)$ & $\begin{array}{l}56(29 ; \\
72)\end{array}$ & $44(36 ; 75)$ \\
\hline \multicolumn{6}{|l|}{$\begin{array}{l}\text { Brain Metastasis prior to other } \\
\text { metastases }\end{array}$} \\
\hline BM only & $\begin{array}{l}19 \\
(33 \%)\end{array}$ & & $5(36 \%)$ & $5(19 \%)$ & $9(53 \%)$ \\
\hline BM after extra cranial mets & $\begin{array}{l}22 \\
(39 \%)\end{array}$ & & $4(28 \%)$ & $13(50 \%)$ & $5(29 \%)$ \\
\hline BM before extra cranial mets & $\begin{array}{l}16 \\
(28 \%)\end{array}$ & & $5(36 \%)$ & $8(31 \%)$ & $3(18 \%)$ \\
\hline \multicolumn{6}{|l|}{ Location of BM } \\
\hline Cerebrum Only & $\begin{array}{l}33 \\
(58 \%)\end{array}$ & & $8(57 \%)$ & $16(62 \%)$ & $10(59 \%)$ \\
\hline Cerebellum Only & $\begin{array}{l}15 \\
(26 \%)\end{array}$ & & $5(36 \%)$ & $8(31 \%)$ & $2(12 \%)$ \\
\hline Multiple locations & $9(16 \%)$ & & $1(7 \%)$ & $2(7 \%)$ & $5(29 \%)$ \\
\hline \multicolumn{6}{|l|}{ Treatment for local Control } \\
\hline None (material from autopsy) & $2(4 \%)$ & & & $1(4 \%)$ & $1(6 \%)$ \\
\hline Surgical resection & $\begin{array}{l}16 \\
(28 \%)\end{array}$ & & $2(14 \%)$ & $10(38 \%)$ & $4(24 \%)$ \\
\hline Surgical Resection followed & $\begin{array}{l}39 \\
(68 \%)\end{array}$ & & $12(86 \%$ & $15(58 \%)$ & $12(70 \%)$ \\
\hline \multicolumn{6}{|l|}{ by radiotherapy } \\
\hline \multicolumn{6}{|l|}{ Number of BM } \\
\hline 1 & $\begin{array}{l}48 \\
(84 \%)\end{array}$ & & $13(93 \%)$ & $23(88 \%)$ & $13(76 \%)$ \\
\hline 2 or more & 9 (16\%) & & $1(7 \%)$ & $3(12 \%)$ & $4(24 \%)$ \\
\hline
\end{tabular}

NGS results fulfilling sequencing quality criteria were obtained for 37 of the 46 matched PT/BM pairs and from 10 of the 11 unmatched BMs. For nine matched pairs, sequencing results were obtained for either only the PT $(n=4)$ or the BM $(n=5)$. In total, NGS results from 41 PTs and 52 BMs including the 37 
matched PT/BM pairs were obtained (Fig. 1.). IHC subtype is reported for the matched 37 PT and BM only.

In the 37 matched PT and BM, receptor status was discordant in eight/ 37 matched pairs (22\%). Three Luminal A tumors had an increase in Ki67 rendering them Luminal B, one Luminal A lost ER receptor expression rendering it TNBC. No Luminal B tumors altered expression. Two HER2/ ER + lost ER receptor, one HER2+/ ER- gained ER receptor. One TNBC gained ER receptor expression. (Fig. 2)

\section{Genetic profile of PT and BM}

Twenty-seven PTs (66\%) and 32 BMs (62\%) exhibited at least one pathogenic mutation (Fig. 3). A pathogenic JAK2 p.V617F mutation in a patient with previously diagnosed myeloproliferative disease was excluded from further analysis. The most commonly mutated gene in the dataset was TP53, with a mutation frequency of $44 \%(20 / 42)$ in PTs and 44\% (25/52) in BMs. PIK3CA mutations were the second most prevalent mutation, with a frequency of $20 \%(9 / 42)$ in PTs and $23 \%(13 / 52)$ in BMs. Of note, a CDH1 mutation was present in a PT/BM pair of lobular BC. Mutations in EGFR, HRAS and RB1 were detected in single PT/BM pairs, as well as, mutations in CDKN2A and PTEN in single samples of PT or BM.

\section{Mutational findings in matched pairs of BM and PT}

In the matched pairs, similarly, the most prevalent mutation was in TP53 in 43\% (16/37). Mutated TP53 was found in three Luminal PTs and four paired BMs (27\%/36\%), eight TNBC PTs and ten paired BMs (47\%/59\%), and six HER2 positive PTs and five paired BMs (67\%/55\%). Mutations in PIK3CA was the second most prevalent mutation in this material. In matched PT/BM pairs, PIK3CA mutations were found in two PTs and four BMs of Luminal B (18\%/36\%); three PTs and BMs in TNBC (18\%/18\%), and in one matched case of HER2 positive tumors (11\%) (Fig. 3, Table 3). The numerical differences of TP53 and PIK3CA mutations in BC subgroups did not reach statistical significance $(p=0.228)(p=0.552)$ respectively.

Table 3. TP53 and PIK3CA mutations in primary breast cancer tumors and brain metastases with NGS results fulfilling sequencing quality criteria. The bottom row shows the concordance in mutation status in the 37 matched brain metastasis and primary breast tumors. 


\begin{tabular}{|c|c|c|c|c|c|}
\hline & $\begin{array}{l}\text { All }(n \\
=41)\end{array}$ & $\begin{array}{l}\text { Luminal A } \\
(n=4)\end{array}$ & $\begin{array}{l}\text { Luminal B } \\
(n=6)\end{array}$ & $\begin{array}{l}\text { TNBC } \\
(n=20)\end{array}$ & $\begin{array}{l}\text { HER2+ } \\
(n=11)\end{array}$ \\
\hline \multicolumn{6}{|l|}{ Primary Tumors $(n=41)$} \\
\hline TP53 & $\begin{array}{l}20 \\
(44 \%)\end{array}$ & $2(40 \%$ & $2(33 \%)$ & $8(40 \%)$ & $4(36 \%)$ \\
\hline \multirow[t]{2}{*}{ PIK3CA } & $\stackrel{9}{(20 \%)}$ & $2(40 \%)$ & $2(33 \%)$ & $5(22 \%)$ & $5(45 \%)$ \\
\hline & $\begin{array}{l}\text { All }(n \\
=52)\end{array}$ & $\begin{array}{l}\text { Luminal A } \\
(n=0)\end{array}$ & $\begin{array}{l}\text { Luminal B } \\
\text { (14) }\end{array}$ & $\begin{array}{l}\text { TNBC } \\
(n=23)\end{array}$ & $\begin{array}{l}\text { HER2+ } \\
(n=15)\end{array}$ \\
\hline \multicolumn{6}{|l|}{ Brain Metastases $(n=52)$ : } \\
\hline TP53 & $\begin{array}{l}25 \\
(44 \%)\end{array}$ & & $4(29 \%)$ & $\begin{array}{l}13 \\
(57 \%)\end{array}$ & $8(53 \%)$ \\
\hline \multirow[t]{2}{*}{ PIK3CA } & $\begin{array}{l}13 \\
(23 \%)\end{array}$ & & $5(36 \%)$ & $5(22 \%)$ & $3(20 \%)$ \\
\hline & $\begin{array}{l}\text { All }(n \\
=37)\end{array}$ & $\begin{array}{l}\text { Luminal A } \\
(n=0)\end{array}$ & $\begin{array}{l}\text { Luminal B } \\
(11)\end{array}$ & $\begin{array}{l}\text { TNBC } \\
(n=17)\end{array}$ & $\begin{array}{l}\text { HER2+ } \\
(n=9)\end{array}$ \\
\hline $\begin{array}{l}\text { Concordance of mutational status in } \\
\text { matched pairs }(n=37) \text { : }\end{array}$ & $\begin{array}{l}29 \\
(67 \%)\end{array}$ & & $7(64 \%)$ & $\begin{array}{l}14 / 17 \\
(82 \%)\end{array}$ & $\begin{array}{l}7 / 9 \\
(78 \%)\end{array}$ \\
\hline
\end{tabular}

\section{Mutational concordance in matched material}

The mutational profile of the genes present in the targeted panel was discordant between the PTs and BMs in nine cases out of 37 (24\%). The mutational differences displayed no discernible pattern. There were three cases of mutations present only in the PT, four with only mutations found in the BM, and one case of mutation in the same gene, but at another position BM. Of note, in two PT/BM pairs, a pathogenic PIK3CA mutation and five TP53 mutations were detected only in the BM.

\section{Survival parameters}

Patients with Luminal and HER2+/ER + BC had the longest BMSS (Table 4) with a median of 28 months (6-64) and 34 months (7-62) respectively, whilst patients with HER2+/ER- and TNBC had BMSS of 11 months $(0-46)$ and eight months $(0-46)$ respectively $(p=0.0851)$. We found equal BMSS when patients with mutations in PIK3CA were compared to patients without PIK3CA mutation ( $p=0.467)$. There was no difference in BMSS when patients with a TP53 mutation were compared with patients without documented TP53 mutation ( $\mathrm{p}=0.456)$. 
Table 4

Survival parameters of the 57 patients with brain metastases analyzed with NGS.

\begin{tabular}{|lllll|}
\hline & $\begin{array}{l}\text { All }(\mathbf{n}= \\
\mathbf{5 7})\end{array}$ & $\begin{array}{l}\text { Luminal B } \\
(\mathbf{n = 1 4})\end{array}$ & $\begin{array}{l}\text { TNBC }(\mathbf{n} \\
=\mathbf{2 6})\end{array}$ & $\begin{array}{l}\text { HER2+ ( } \mathbf{~} \\
=\mathbf{1 7})\end{array}$ \\
\hline $\begin{array}{l}\text { Mean (SD) Brain Metastasis Specific Survival } \\
\text { (months) }\end{array}$ & $\mathbf{1 7}(\mathbf{1 6 . 9})$ & $28(18.9)$ & $9(7.4)$ & $21(19.8)$ \\
\hline $\begin{array}{l}\text { Median (min; max) Brain Metastasis Specific } \\
\text { Survival (months) }\end{array}$ & $\begin{array}{l}\mathbf{1 2 . 5 ( 0 ;} \\
\mathbf{6 4})\end{array}$ & $28(6 ; 64)$ & $8(1 ; 25)$ & $13(0 ; 62)$ \\
\hline Mean (SD) Overall Survival (months & $\begin{array}{l}\mathbf{6 9} \\
\mathbf{( 5 2 . 3 )}\end{array}$ & $98(41)$ & $57(60.7)$ & $66(33.4)$ \\
\hline Median (min; max) Overall Survival (months) & $\begin{array}{l}\mathbf{5 0 ( 1 0 ;} \\
\mathbf{2 8 8})\end{array}$ & $91(35 ; 177)$ & $\begin{array}{l}40(10 ; \\
288)\end{array}$ & $\begin{array}{l}96(42 ; \\
107)\end{array}$ \\
\hline
\end{tabular}

\section{Discussion}

Concordant with previous published data, we confirm that TP53 and PIK3CA is the most common mutations in BCBM, as well as, the matched PTs, in to our very best knowledge so far one of the largest cohorts of genetically characterized BCBM [16-21]. Difficulties in accessing intra-cranial tissue samples have hampered development of systemic treatments that could have an effect in the treatment of patients with BMs. Our cohort, consists of 52 analyzed cases, and 37 cases with matched PT, all from one region in Sweden. The difficulty in obtaining tissue from the brain results in few tumors which limits the study when comparing BC subgroups or in survival analyses. Although the tissue material is partly old, with the first samples collected in 1994, sequencing results by NGS were successfully obtained in $90 \%$ of the BMs. At least one mutation, among 50 cancer driver genes, was present in $62 \%$ of the analyzed samples which is in the similar range as reported in previous publications [16-21]. Concordant with these previous studies, we found TP53 and PIK3CA as the most commonly mutated driver genes, both in the BMs and PTs. Of these two mutations, PIK3CA are considered targetable, whilst TP53 mutations can be indirectly attacked through restoration of the transcriptional activity resulting in a functional wild-type TP53 protein [22, 23].

The drug alpelisib, is a PI3Ka-specific inhibitor available for patients with recurrent PIK3CA mutated Luminal/HER2 negative BC [23]. Previous published data show that approximately $40 \%$ of ER positive PT harbor a PIK3CA mutation [24]. Two studies that compared PIK3CA mutations in PTs and BC metastases reported mutations in $33 \%$ of PTs and $30 \%$ of metastases and $45 \%$ of PTs and $53 \%$ in metastases respectively $[18,25]$. There were few or no patients with BMs in the above mentioned studies. Our figures in BCBM were lower with a PIK3CA mutation frequency of $20 \%$ in PTs and $23 \%$ in the BMs. A recently published systematic review compared $164 \mathrm{BMs}$ with its matched PT in 126 patients extracted from 13 studies and found PIK3CA mutations in $22 \%$ of the patients with BMs which in line with our results [26]. It might be hypothesized that PIK3CA mutations is lower in BMs compared to other metastatic sites, however, additional studies are required to adequately answer this question. As expected, we found the highest proportion of PIK3CA mutations in Luminal BC. Of note, in our material two out of eight 
pathogenic PIK3CA mutations and five TP53 mutations were found in the BMs only and not in the PT underlining the need for re-evaluation of metastatic tissue or possibly by analysis of cell free DNA (cfDNA) [27].

We found PIK3CA mutations as the second most common mutation after TP53 in matched pairs of TNBC with $18 \%$ PIK3CA mutations all concordant in PTs and BMs. This is in line with previous published data in which PIK3CA mutations was the second most common mutation after TP53, especially in basallike and luminal androgen receptor subtypes of TNBC [28-30]. The combined treatment of alpelisib and nab-paclitaxel is currently under investigation in pre-treated TNBC with either loss of PTEN expression or a PIK3CA mutation (NCT04251533).

The prognostic role of PIK3CA mutations in HER2 $+\mathrm{BC}$ has been extensively investigated. Mutations in PIK3CA has been associated with less effect of HER2 blocking therapy, both in the neo-adjuvant setting, and for recurrent $B C$ in terms of lower $\mathrm{PCR}$ rates and shorter survival respectively [31, 32]. We found PIK3CA mutations in only $10 \%$ of the HER $2+$ cases, which is lower than the previously reported frequency of $20-40 \%$. [24]. The limited number of HER2 + patients in our cohort is considered to be the cause of this discrepancy. The effect of alpelisib in HER2 + breast cancer is under investigation in one ongoing and one completed clinical trial (NCT02038010; NCT04208178).

Almost $50 \%$ of the PTs in the present study population that have a high proportion of TNBC harbored a TP53 mutation, in contrast to the 20-35\% mutation prevalence reported in unselected primary BC [33]. The Cancer Genome Atlas reveals an enrichment of TP53 mutations in basal-like and HER2 enriched BC [24]. Interestingly, previous data reveal that a high proportion of patients with a TP53 mutation in the primary BC developed BM $[34,35]$. We lack data on the molecular subtypes in our material, but find an increased number of TP53 mutations in TNBC and HER2/ER- BC. Enrichment of TP53 mutations in BMs was not seen in the HER2 amplified subgroup, a finding that must be interpreted with caution due to the very small sample size of the HER2 group. Other detected mutations in our series, CDH1, EGFR, HRAS, $R B 1, C D K N 2 A$ and $P T E N$ were rare, in general found in single or both samples from one patient.

Roughly $25 \%$ of the matched pairs changed $\mathrm{IHC}$ based $\mathrm{BC}$ subtype in the BMs. The most common change was from Luminal A in PT to other subtypes, in most cases Luminal B in BMs. This is in accordance with previous results in which 219 patients showed a $36 \%$ overall discordance with the most common change in form of loss of $\mathrm{PgR}$ [36]. In a review pooling a total of 3384 matched pairs of $\mathrm{BC}$ and metastases from all organs, BM showed a discordant median rate of $22 \%$ compared to $45 \%$ in liver metastases and $16 \%$ in lymph node metastasis [37]. TNBC was the most stable group with less than $10 \%$ showing a gain in ER (one case) and HER2 (one case).

A limitation of our study is that genetic profiling was performed using a relatively small NGS panel of 50 genes. Even though the panel gives broad coverage of important cancer driver genes, more comprehensive sequencing might have provided further details in the landscape of mutation discordancy between PTs and BMs. Still, we believe that the relatively large number of matched PT and BM add to the knowledge about the biology of BMs, how the metastatic process affects actionable genes. 
In conclusion, we confirm mutations in TP53 and PIK3CA to be common in both primary breast tumors and BMs but the proportion varied depending on the subgroup. Mutation pattern, as well as IHC based subtypes were discordant in approximately $25 \%$ of the patients underlining the need for re-biopsy at disease progression. In this context, analysis of cfDNA may be a fruitful avenue in patients with BMs, placing hope in promising results with liquid biopsy from $\operatorname{CSF}[27,38,39]$.

\section{Declarations}

Funding: This study was supported by grants from the Swedish Breast Cancer Association (Bröstcancerförbundet), the Swedish State under the LUA-agreement (Sahlgrenska University Hospital, Gothenburg) (VGFOUREG-667831).

Conflict of Interests: The authors declare no conflicts of interest.

Ethical Approval: The study has been conducted in accordance with the Declaration of Helsinki and the Sahlgrenska University Hospital Ethical Review Board, Gothenburg, Sweden approved the study (Swedish Ethical Review Authority, 460-09, T592-14), which also waived the need for informed consent due to the retrospective design. Approval for chart studies was approved by the departments of Surgery and Oncology at Sahlgrenska University Hospital, the department of Surgery at Näl Hospital, the department of Surgery at Halmstad Hospital, the department of Surgery at SKAS, and the department of surgery at SÄS.

Data disclosure: All pathological and uncertain mutations are added as a supplemental table. Clinical data is not included as this would allow for possibly identifying individuals.

Consent for publication: Not applicable

Author Contributions: $\mathrm{BL}, \mathrm{AT}$, and FE conceived of the study. CA, SDL, AK, ER, FE, and BL developed the methodology for the study. CA, ER, SDL, CC, AS, AK, FE and AT participated in data acquisition. AT, FE, HF, and $B L$ participated in data analysis and interpretation. AT, BL, and HF wrote and/ or edited the manuscript. All authors read and approved the final manuscript.

\section{References}

1. Lin, N.U., J.R. Bellon, and E.P. Winer, CNS metastases in breast cancer. J Clin Oncol, 2004. 22(17): p. 3608-17.

2. Witzel, I., et al., Breast cancer brain metastases: biology and new clinical perspectives. Breast Cancer Res, 2016. 18(1): p. 8.

3. Niikura, N., et al., Treatment outcomes and prognostic factors for patients with brain metastases from breast cancer of each subtype: a multicenter retrospective analysis. Breast Cancer Res Treat, 2014. 147(1): p. 103-12. 
4. Witzel, I., et al., Treatment and outcomes of patients in the Brain Metastases in Breast Cancer Network Registry. European Journal of Cancer, 2018. 102: p. 1-9.

5. Berghoff, A., et al., Brain metastases free survival differs between breast cancer subtypes. $\mathrm{Br} \mathrm{J}$ Cancer, 2012. 106(3): p. 440-6.

6. Thulin, A., et al., Clinical outcome of patients with brain metastases from breast cancer - $A$ population based study over 21 years. Breast, 2020. 50: p. 113-124.

7. Bartsch, R., et al., Impact of anti-HER2 therapy on overall survival in HER2-overexpressing breast cancer patients with brain metastases. Br J Cancer, 2012. 106(1): p. 25-31.

8. Brufsky, A.M., et al., Central nervous system metastases in patients with HER2-positive metastatic breast cancer: incidence, treatment, and survival in patients from registHER. Clin Cancer Res, 2011. 17(14): p. 4834-43.

9. Gerstner, E.R. and R.L. Fine, Increased permeability of the blood-brain barrier to chemotherapy in metastatic brain tumors: establishing a treatment paradigm. J Clin Oncol, 2007. 25(16): p. 2306-12.

10. Yeung, C., et al., Estrogen, progesterone, and HER2/neu receptor discordance between primary and metastatic breast tumours-a review. Cancer Metastasis Rev, 2016. 35(3): p. 427-37.

11. Cagney, D.N., et al., Incidence and prognosis of patients with brain metastases at diagnosis of systemic malignancy: a population-based study. Neuro Oncol, 2017. 19(11): p. 1511-1521.

12. del Rivero, J. and E.C. Kohn, PARP Inhibitors: The Cornerstone of DNA Repair-Targeted Therapies. Oncology (Williston Park), 2017. 31(4): p. 265-73.

13. Murthy, R., et al., Tucatinib with capecitabine and trastuzumab in advanced HER2-positive metastatic breast cancer with and without brain metastases: a non-randomised, open-label, phase $1 \mathrm{~b}$ study. Lancet Oncol, 2018. 19(7): p. 880-888.

14. Andre, F., et al., Alpelisib for PIK3CA-Mutated, Hormone Receptor-Positive Advanced Breast Cancer. N Engl J Med, 2019. 380(20): p. 1929-1940.

15. Bertucci, F., et al., Comparative genomic analysis of primary tumors and metastases in breast cancer. Oncotarget, 2016. 7(19): p. 27208-19.

16. Muller, K.E., et al., Targeted next-generation sequencing detects a high frequency of potentially actionable mutations in metastatic breast cancers. Experimental and Molecular Pathology, 2016. 100(3): p. 421-425.

17. Lee, J.Y., et al., Mutational profiling of brain metastasis from breast cancer: matched pair analysis of targeted sequencing between brain metastasis and primary breast cancer. Oncotarget, 2015. 6(41): p. 43731-42.

18. Fumagalli, C., et al., Inter-tumor genomic heterogeneity of breast cancers: comprehensive genomic profile of primary early breast cancers and relapses. Breast Cancer Research, 2020. 22(1).

19. Lee, J.Y., et al., Gene Expression Profiling of Breast Cancer Brain Metastasis. Sci Rep, 2016. 6: p. 28623. 
20. Iwamoto, T., et al., Distinct gene expression profiles between primary breast cancers and brain metastases from pair-matched samples. Sci Rep, 2019. 9(1): p. 13343.

21. Schrijver, W., et al., Mutation Profiling of Key Cancer Genes in Primary Breast Cancers and Their Distant Metastases. Cancer Res, 2018. 78(12): p. 3112-3121.

22. Lehmann, S., et al., Targeting p53 in vivo: a first-in-human study with p53-targeting compound APR246 in refractory hematologic malignancies and prostate cancer. J Clin Oncol, 2012. 30(29): p. 36339.

23. André, F., et al., Alpelisib for PIK3CA-Mutated, Hormone Receptor-Positive Advanced Breast Cancer. New England Journal of Medicine, 2019. 380(20): p. 1929-1940.

24. Koboldt, D.C., et al., Comprehensive molecular portraits of human breast tumours. Nature, 2012. 490(7418): p. 61-70.

25. Dupont Jensen, J., et al., PIK3CA mutations may be discordant between primary and corresponding metastatic disease in breast cancer. Clin Cancer Res, 2011. 17(4): p. 667-77.

26. Morgan, A.J., A. Giannoudis, and C. Palmieri, The genomic landscape of breast cancer brain metastases: a systematic review. Lancet Oncol, 2021. 22(1): p. e7-e17.

27. Kodahl, A.R., et al., Correlation between circulating cell-free PIK3CA tumor DNA levels and treatment response in patients with PIK3CA-mutated metastatic breast cancer. Molecular Oncology, 2018. 12(6): p. 925-935.

28. Bareche, Y., et al., Unravelling triple-negative breast cancer molecular heterogeneity using an integrative multiomic analysis. Ann Oncol, 2018. 29(4): p. 895-902.

29. Shah, S.P., et al., The clonal and mutational evolution spectrum of primary triple-negative breast cancers. Nature, 2012. 486(7403): p. 395-399.

30. Mosele, F., et al., Outcome and molecular landscape of patients with $<e m>P I K 3 C A</ e m>-$ mutated metastatic breast cancer. Annals of Oncology, 2020. 31(3): p. 377-386.

31. Baselga, J., et al., Biomarker analyses in CLEOPATRA: a phase III, placebo-controlled study of pertuzumab in human epidermal growth factor receptor 2-positive, first-line metastatic breast cancer. J Clin Oncol, 2014. 32(33): p. 3753-61.

32. Loibl, S., et al., PIK3CA mutations are associated with lower rates of pathologic complete response to anti-human epidermal growth factor receptor 2 (her2) therapy in primary HER2-overexpressing breast cancer. J Clin Oncol, 2014. 32(29): p. 3212-20.

33. Linderholm, B.K., et al., The expression of vascular endothelial growth factor correlates with mutant p53 and poor prognosis in human breast cancer. Cancer Res, 2001. 61(5): p. 2256-60.

34. Ding, L., et al., Genome remodelling in a basal-like breast cancer metastasis and xenograft. Nature, 2010. 464(7291): p. 999-1005.

35. Powell, E., D. Piwnica-Worms, and H. Piwnica-Worms, Contribution of p53 to metastasis. Cancer discovery, 2014. 4(4): p. 405-414. 
36. Hulsbergen, A.F.C., et al., Subtype switching in breast cancer brain metastases: a multicenter analysis. Neuro-oncology, 2020. 22(8): p. 1173-1181.

37. Yeung, C., et al., Estrogen, progesterone, and HER2/neu receptor discordance between primary and metastatic breast tumours-a review. Cancer and Metastasis Reviews, 2016. 35(3): p. 427-437.

38. Siravegna, G., et al., Genotyping tumour DNA in cerebrospinal fluid and plasma of a HER2-positive breast cancer patient with brain metastases. ESMO Open, 2017. 2(4).

39. Keup, C., et al., Longitudinal Multi-Parametric Liquid Biopsy Approach Identifies Unique Features of Circulating Tumor Cell, Extracellular Vesicle, and Cell-Free DNA Characterization for Disease Monitoring in Metastatic Breast Cancer Patients. Cells, 2021. 10(2).

\section{Figures}




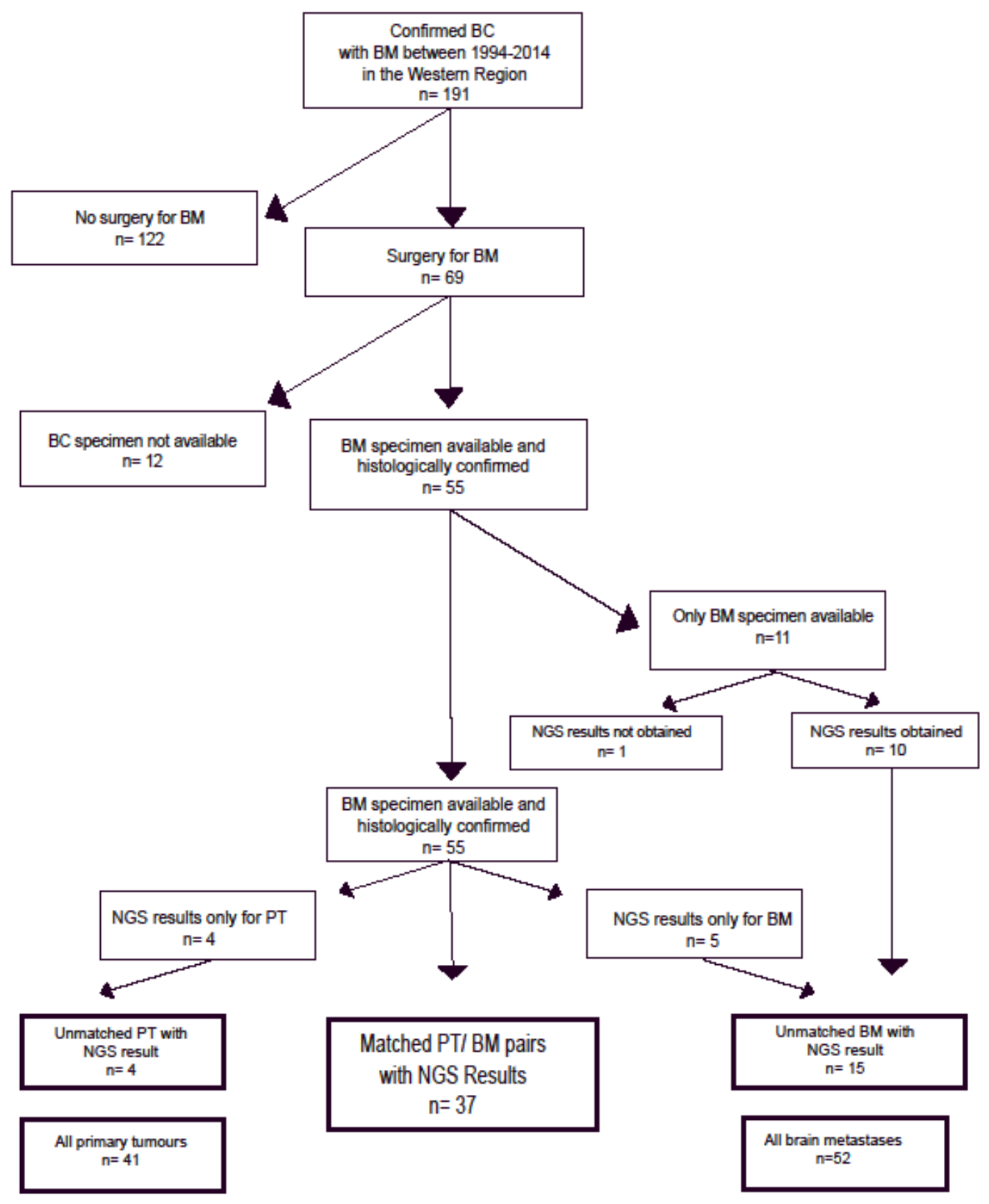

\section{Figure 1}

Consolidated Standards of Reporting Trials (CONSORT) diagram describing the identification process of the study population showing the final numbers of brain metastasis, primary breast tumors and matched pairs of brain metastasis and primary breast tumors. 


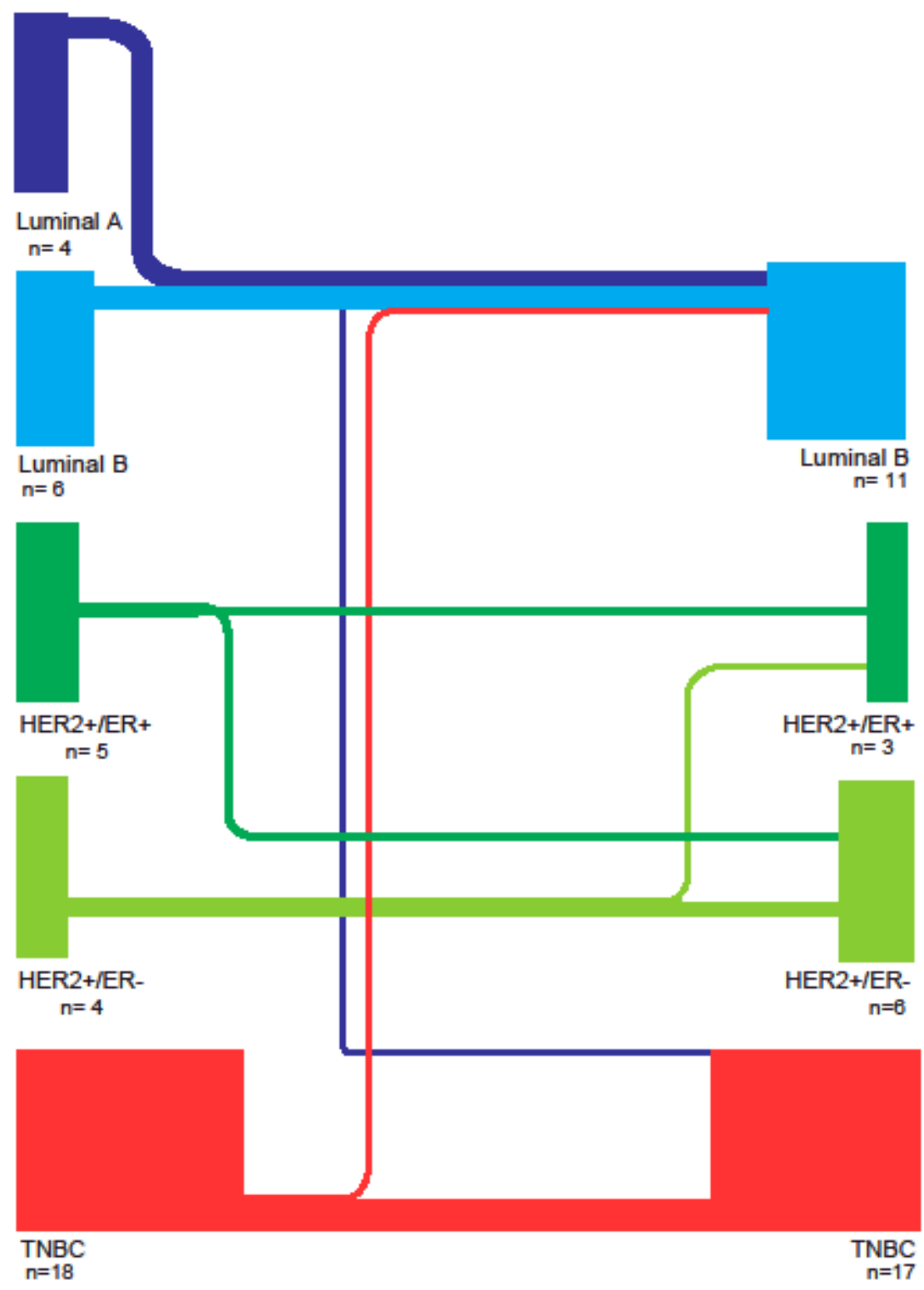

Figure 2

Breast cancer subtype according to expression of steroid receptors, HER2 and Ki67 in primary breast tumors and brain metastases. The thickness of the line represents the number of tumors that changed subgroup in respective group. 

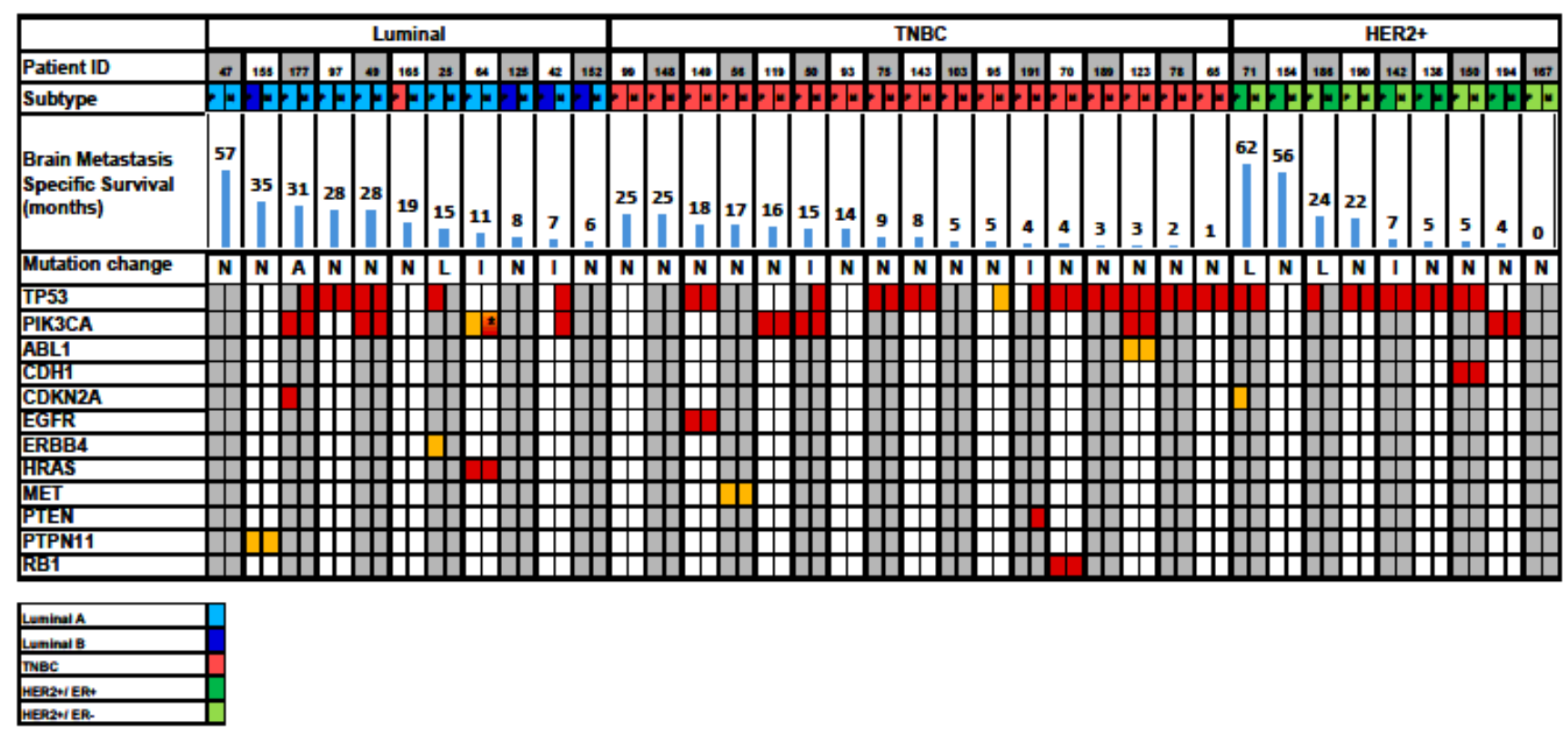

Presogencementation

Unoertain peneopelicty

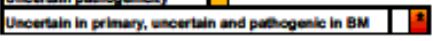

\section{Figure 3}

All matched pairs of brain metastasis and primary breast tumors color coded into subgroups with pathological and uncertain mutations shown. Brain metastasis specific survival (months) for each patient is shown in blue bars above the mutations. 